\title{
Paracrine osteoprotegerin and $\beta$-catenin stabilization support synovial sarcomagenesis in periosteal cells
}

\author{
Jared J. Barrott, ${ }^{1}$ Benjamin E. Illum, ${ }^{2}$ Huifeng Jin, ${ }^{1}$ Matthew L. Hedberg, ${ }^{3}$ Yanliang Wang, ${ }^{1}$ Allie Grossmann, ${ }^{4}$ Malay Haldar, ${ }^{5}$ \\ Mario R. Capecchi, ${ }^{2}$ and Kevin B. Jones' \\ 'Departments of Orthopaedics and Oncological Sciences, and 'Department of Human Cenetics, University of Utah, Salt Lake City, Utah, USA. ${ }^{3}$ Department of Pathology and Immunology, Washington \\ University, St. Louis, Missouri, USA. ${ }^{4}$ Department of Pathology, University of Utah, Salt Lake City, Utah, USA. ${ }^{5}$ Department of Pathology, University of Pennsylvania, Philadelphia, Pennsylvania, USA.
}

\begin{abstract}
Synovial sarcoma (SS) is an aggressive soft-tissue sarcoma that is often discovered during adolescence and young adulthood. Despite the name, synovial sarcoma does not typically arise from a synoviocyte but instead arises in close proximity to bones. Previous work demonstrated that mice expressing the characteristic SS18-SSX fusion oncogene in myogenic factor 5-expressing (Myf5-expressing) cells develop fully penetrant sarcomagenesis, suggesting skeletal muscle progenitor cell origin. However, Myf5 is not restricted to committed myoblasts in embryos but is also expressed in multipotent mesenchymal progenitors. Here, we demonstrated that human SS and mouse tumors arising from SS18-SSX expression in the embryonic, but not postnatal, Myf5 lineage share an anatomic location that is frequently adjacent to bone. Additionally, we showed that SS can originate from periosteal cells expressing SS18-SSX alone and from preosteoblasts expressing the fusion oncogene accompanied by the added stabilization of $\beta$-catenin, which is a common secondary change in SS. Expression and secretion of the osteoclastogenesis inhibitory factor osteoprotegerin enabled early growth of SS18SSX2-transformed cells, indicating a paracrine link between the bone and synovial sarcomagenesis. These findings explain the skeletal contact frequently observed in human SS and may provide alternate means of enabling SS18-SSX-driven oncogenesis in cells as differentiated as preosteoblasts.
\end{abstract}

\section{Introduction}

Synovial sarcoma (SS) is the most common soft-tissue sarcoma diagnosed during adolescence and young adulthood (1). SS consistently bears a $t(X ; 18)$ chromosomal translocation, which produces 1 of 3 possible fusion oncogenes: SS18-SSX1, SS18-SSX2, or SS18$S S X 4$ (2). No additional consistent genomic perturbations have been identified by profiling, suggesting that the fusion oncogene can drive transformation independently (3-5).

The transforming impact of an SS18-SSX fusion oncoprotein is profound, resulting in a germ layer cell-fate change in many SSs, such that, despite mesodermal origins, SS cells develop epithelial characteristics of endoderm and can even achieve a tissue morphology that includes mucinous gland formation, termed biphasic SS (6). This feature may have helped to keep the cell of origin for SS an enigma. While SS18-SSX1 and SS18$S S X 2$ have each proven sufficient to initiate fully penetrant (on a per-animal basis) synovial sarcomagenesis when expressed in specific cell lineages in mice, most cell lineages cannot tolerate the expression of either fusion oncogene. The original mouse model identified the Myf5Cre lineage as permissive to synovial sarcomagenesis (7). This was interpreted to indicate a myoblast cell of origin. However, the specific Myf5 IRES-Cre-knockin allele used was found on other lineage-tracing experiments to

Conflict of interest: The authors have declared that no conflict of interest exists

Submitted: May 4, 2017; Accepted: October 10, 2017.

Reference information: J Clin Invest. 2018;128(1):207-218.

https://doi.org/10.1172/JCI94955. have broader multipotency, with the lineage traced to osteoblasts and chondrocytes, as well as the expected myocytes $(8,9)$.

Among soft-tissue sarcomas, SS also has a unique relationship with the skeleton and osteogenesis. A close proximity to bones has been described as a common characteristic of SS $(10,11)$. SS is also one of very few soft-tissue sarcomas that can produce mineralized bone matrix within the tumor mass (12). Although this bone matrix is typically present in a small minority of the volumetric substance of an SS, an "ossifying" variant of SS, with abundant bone matrix dominating the tumor volume, has also been described (13-17).

In previous experiments, we combined the expression of SS18SSX2 with genetic stabilization of $\beta$-catenin (CTNNB1) in a localized tumorigenesis model induced by injection of AdCre virus or TATCre protein (18). We pursued these experiments because of the observation of $A P C$-inactivating and CTNNB1-stabilizing mutations in a subset of human SSs, with a larger portion exhibiting deregulation of Wnt signaling (19-22). While the dramatic sarcomagenesis that resulted in the combination-genotype mice reflected this subset of human SSs that share overactive Wnt signaling, it also provided the first mouse model information relating SS to the skeleton, as these tumors were directly juxtaposed to and, by growth, destroyed adjacent bone structures. This apparent invasion suggested either that cells within the bone were transformed by SS18-SSX with the help of $\beta$-catenin stabilization, or that bones offered a welcoming microenvironment for invasive growth of SS cells.

Differentially expressed genes in these $\beta$-catenin-stabilized tumors, compared with mouse SSs, which lacked genetic stabilization of $\beta$-catenin, supported these 2 hypotheses. First, $\beta$-catenin- 
A

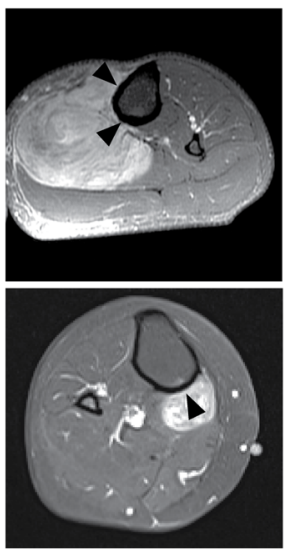

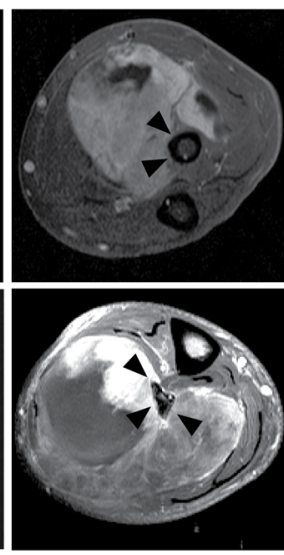

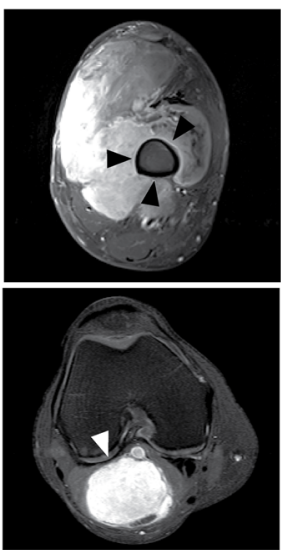

B

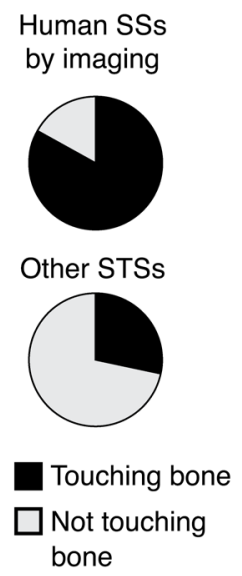

Human SSs

by imaging

Figure 1. Synovial sarcomagenesis occurs in proximity to bone. (A) Representative MRIs demonstrate the relationship of human SSs to bone in 5 patients with tumors directly abutting the bone surface (black arrowheads) and 1 patient in whom the tumor did not touch the bone directly (white arrowhead), abutting instead the knee joint capsule. (B) Pie charts demonstrating the prevalence of neoplasms touching bone among human SSs $(n=48)$ and neoplasms near bone (within 1 centimeter) among non-SS human soft-tissue sarcomas (STSs) of the extremities $(n=28)$. (C) MRIs demonstrating SSs (solid white arrowheads) and adjacent bones (empty white arrowheads) in Myf5Cre hSS2 mice, as well as (D) H\&E-stained histology images from the same, demonstrating tumor cells abutting, involving, or replacing periosteum (Black arrowheads indicate the periosteal surface with tumor involvement. Open arrowheads indicate the endosteal surface and marrow space.). Scale bars: $100 \mu \mathrm{m}$. (E) Pie chart demonstrating the prevalence of tumor cells touching the bone surface in random sections of tumors from 8 mice ( $n=33$ tumors).
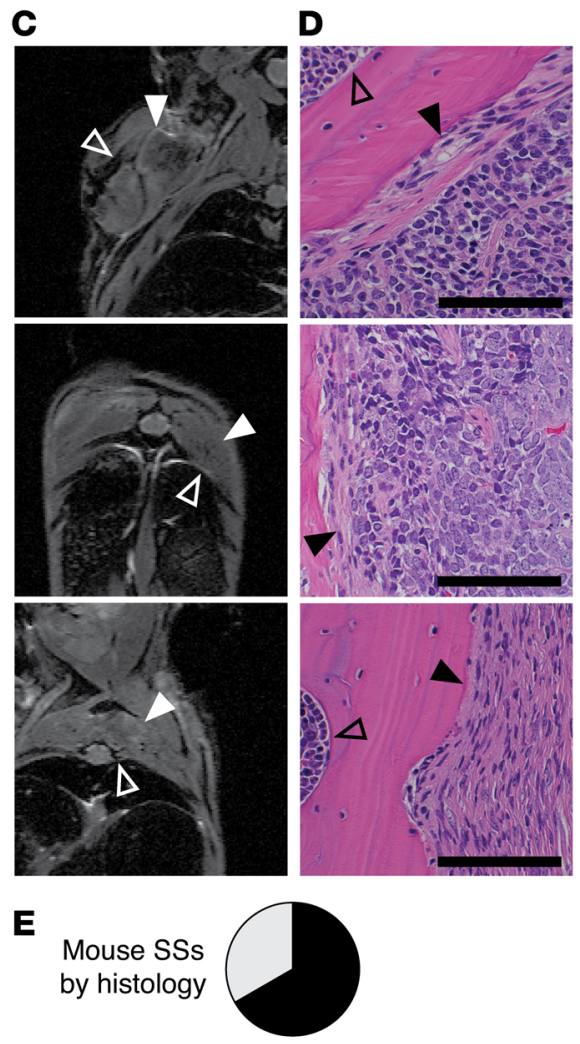

stabilized tumors expressed higher levels of mesenchyme- and osteogenesis-related genes. This does not explicitly implicate, but may suggest, that these tumors derived from more bone-oriented cells of origin. Second, they also expressed more Opg (also known as Tnfrsf $11 b$, which codes for osteoprotegerin, a secreted factor), which raised the possibility of microenvironmental effects of the bone. $\beta$-Catenin has been shown to drive the expression of OPG in osteoblasts $(23,24)$, the expression and secretion of which will shut down osteoclastogenesis as a RANKL dummy receptor $(25,26)$. Notably, OPG can also function as a dummy receptor for TRAIL, preventing extrinsic apoptosis (27). OPG expression is variable and generally low across reported human SS transcriptomes (28), and the elevated Opg expression observed in the group of $\beta$-catenin-stabilized mouse tumors could not be unambiguously attributed to tumor cells, as stromal cells or reactive bone tissue cells within the tumor tissue might alternatively account for its expression. However, as a secreted protein, OPG could function in a paracrine fashion to provide a sarcomagenesis-enabling environment. We sought to study the relationship between synovial sarcomagenesis and the skeleton by testing each of these hypotheses more thoroughly through genetic experiments in the mouse models.

\section{Results}

Synovial sarcomas appear in close proximity to bone. To determine whether a location adjacent to bone was relatively more common in SSs compared with other soft-tissue sarcomas, we analyzed a consecutive series of 48 human SSs. We observed that 40 of these SSs appeared to be directly juxtaposed to bone, without intervening periosteum (Supplemental Table 1; supplemental material available online with this article; https://doi.org/10.1172/
JCI94955DS1, and Figure 1, A and B). Of the 8 SSs that were not juxtaposed to bone, 4 arose adjacent to the knee joint capsule (Supplemental Table 1 and Figure 1A), a structure similar to that of the periosteum in embryogenesis (29). Further, 6 of the 8 SSs that did not involve periosteal tissues had bone matrix production within their substance (Supplemental Table 1). In contrast, a control group of 28 nonretroperitoneal, consecutive soft-tissue sarcomas of nonsynovial histologic type included only 8 that even approached proximity to the skeleton $\left(P<5 \times 10^{-7}\right.$, Fisher's exact 2-tailed analysis of these ratios; Supplemental Table 1 and Figure 1B). A second control cohort of 28 consecutive soft-tissue sarcomas of only pleiomorphic histology included 3 with growth in close proximity to the skeleton (Supplemental Table 1).

This anatomic location and proximity to bone was also recapitulated in our genetically engineered mouse models of SS. When we conditionally activated the Rosa26-LSL-SS18-SSX2-IRESeGFP ( $h S S 2$ ) allele in the Myf5Cre lineage, we frequently detected SSs next to bone, with the most common locations being in the extremities and the rib cage (Figure 1, C-E, and Supplemental Figure 1A).

We previously observed that TATCre injections into peritibial tissues consistently produced tumors in $h S S 2 C t n n b 1^{e \times 3 f f / W T}$ mice, which express a floxed Ctnnb1 exon 3 (18). To test the tumor-originating capacity of other mesenchymal tissue compartments, we injected 8 hSS2 Ctnnb1 ${ }^{e x 3 f / W T}$ littermate mice with $10 \mu$ TATCre to delete Ctnnb1 exon 3 in cells within the tibialis anterior muscle adjacent to bone, in the contralateral quadriceps muscle distant from bone, and in the subcutaneous tissue of the abdominal wall. By 8 weeks after injection, each of the peritibial locations produced a tumor (Supplemental Figure 1B); none of the subcutaneous-only injections produced tumors (Supplemental Figure 1C); 
A
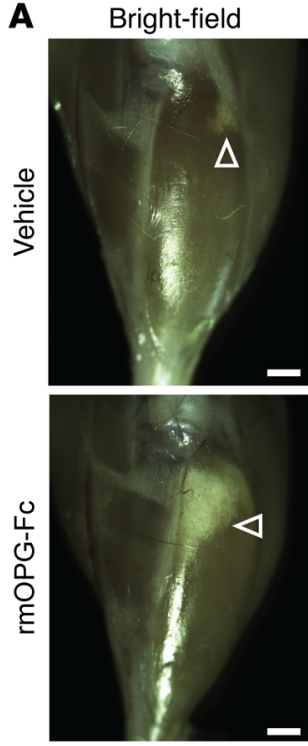

GFP
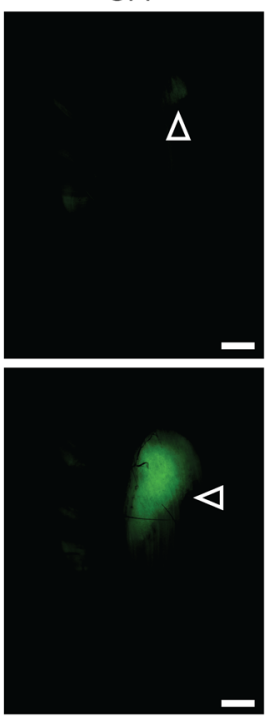

B

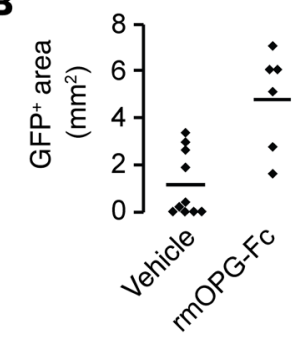

C

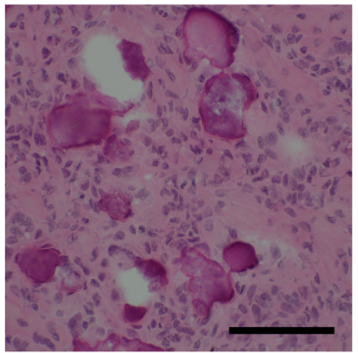

D

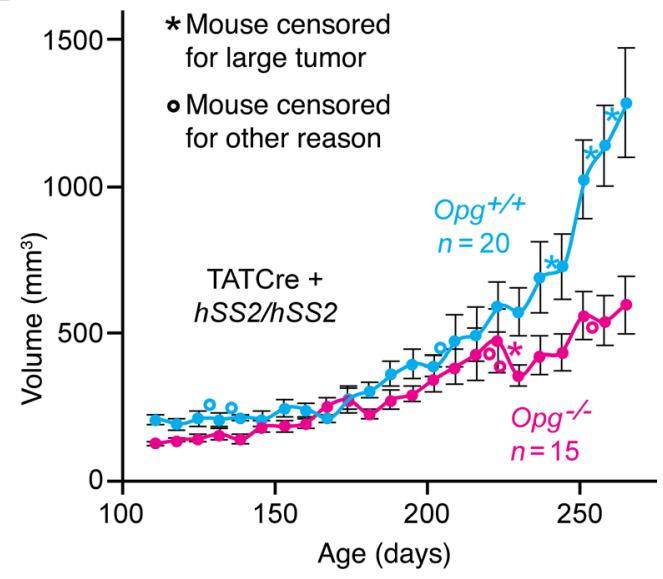

Figure 2. Osteoprotegerin enhances early synovial sarcomagenesis but is not critical to it. (A) Gross light and GFP fluorescence micrographs from hSS2 heterozygous mice that developed appreciably transformed tissue (white arrowheads) by 6 weeks of age, after injection into the anterior compartment of the leg at 4 weeks of age of TATCre, followed by vehicle or rmOPG-Fc, every 48 hours until harvest. Scale bars: 1 mm. (B) Plot of the 2D area of persistent GFP fluorescence following the vehicle control or rmOPG-Fc injections (bars indicate the mean; $n=$ at least 6 per group; $P=0.0066$, by 2 -tailed Student's $t$ test). (C) H\&E histology photomicrograph representative of surviving GFP ${ }^{+}$tissue in either group. Scale bar: $100 \mu \mathrm{m}$. (D) Growth curves of tumors in $h S 52$ homozygous mice with noted Opg genotypes, injected with TATCre at 4 weeks of age. Data represent the mean \pm SEM. $P=0.04$, by ANOVA; $P<0.04$, by 2-tailed Student's $t$ test for comparisons of mice aged $\geq 244$ days.

and 5 of the 8 quadriceps muscle injections produced tumors, 3 of which developed adjacent to the posterior femur (Supplemental Figure 1, D-F). These data cannot prove that cells with osteogenic differentiation potential are the cells of origin for these tumors, but they suggest that synovial sarcomagenesis has an unusual predilection for growing near the skeleton.

Osteoprotegerin can enable synovial sarcomagenesis, but is not necessary for it. To test the potential contribution of paracrine OPG to synovial sarcomagenesis, we locally administered recombinant mouse OPG (rmOPG) every other day (or vehicle control) to 4-week-old hSS2 mice following TATCre injection into the tibialis anterior muscle. Compared with the vehicle control injections, rmOPG significantly enhanced the survival of GFP-fluorescent, SS18-SSX2-expressing cells 2 weeks after TATCre injection (Figure $2, \mathrm{~A}-\mathrm{C}$ ), suggesting a role for paracrine OPG in enabling synovial sarcomagenesis at an early stage.

To test the impact of genetically removing Opg gene function, we bred mice to be WT, heterozygous, or homozygous for a spontaneous loss-of-function mutant allele of $O p g$ as well as heterozygous for both $M y f 5 C r e$ and $h S S 2$. These were difficult experiments to perform, given that Myf5Cre hSS2 mice are already developmentally challenged, with severe kyphosis and weight loss that accompanies, but are independent of, tumorigenesis and likely results from $M y f 5$ lineage loss $(5,7,8)$. The added developmental side effects of lost OPG function led to the early death of the combination mutant mice as a result of morbidity that was also not tumor related (Supplemental Figure 2).

We repeated the experiment in mice via localized induction of SS by TATCre injection into homozygous $h S S 2$ mice that were either homozygously WT or homozygously mutant for $O p g$. These mice lack the developmental side effects of $M y f 5 \mathrm{Cre}$ lineage transformation. These locally induced tumors developed more slowly in the mice lacking OPG (Figure 2D).

Embryonic Myf5Cre-expressing cells, but not postnatal myoblasts, can give rise to SS. To test whether postnatal myoblasts expressing Myf 5 Cre could give rise to SSs, an allele with SS18-SSX2 under the control of the TetO promoter, and separated from it by a floxed stop cassette, was targeted to a noncoding region in the RNAPolII locus (this allele was termed hSS2T) (Figure 3A). Fourteen days postcoitum, embryonic fibroblasts, which were heterozygous for both the $h S S 2 T$ allele and an allele that places the reverse tet transactivator (rtTA) in the Rosa26 locus behind a floxed stop (30), were harvested and recombined in vitro by the application of TATCre or vehicle and exposed to either doxycycline or saline control. These cells demonstrated a mild GFP signal from recombination of the $r t T A$ allele, but stronger GFP fluorescence from the activation of the $h S S 2 T$ allele after TATCre application and in the presence of doxycycline (Figure 3B).

Next, we bred mice to be triple-heterozygous for $\mathrm{Myf} 5 \mathrm{Cre}$, the conditional $r t T A$ allele, and the $h S S 2 T$ allele. Cohorts of these mice were provided drinking water with $0,1,2$, or $6 \mathrm{mg} / \mathrm{ml}$ doxycycline and $5 \%$ sucrose continuously after weaning ( 3 weeks of age). We maintained these mice for over a year and found that no tumors had developed (Figure 3C; $n>25$ per dose group). For a second cohort of mice, the parents were bred with continuous doxycycline exposure at the same doses (but therefore beginning from conception for the experimental mice). No pups were born to the breeding pairs exposed to the $6-\mathrm{mg} / \mathrm{ml}$ dose of doxycycline. Only 1 litter of 5 pups was born to 1 of the pairs exposed to $2 \mathrm{mg} / \mathrm{ml}$ doxycycline. Each of these mice formed tumors over the course of 1 year of continuous 
A
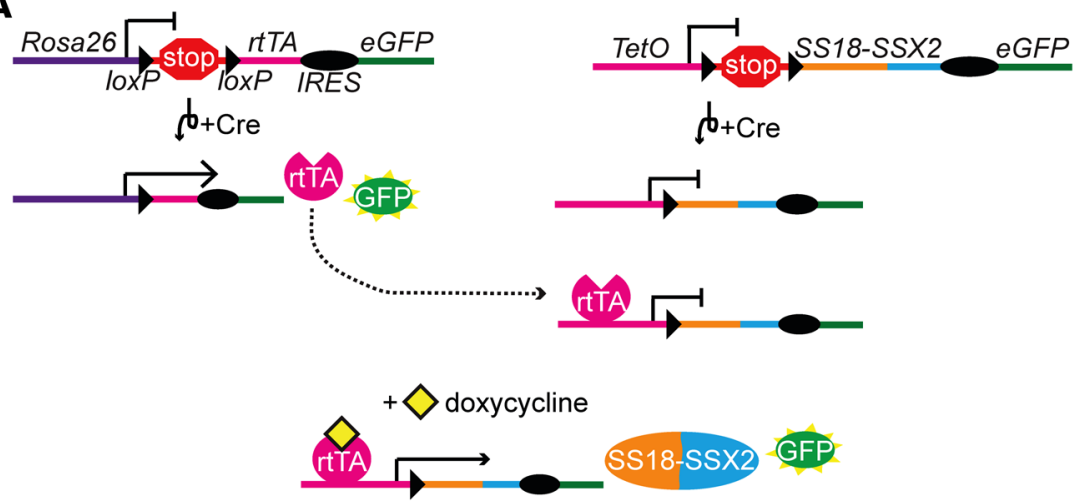

B
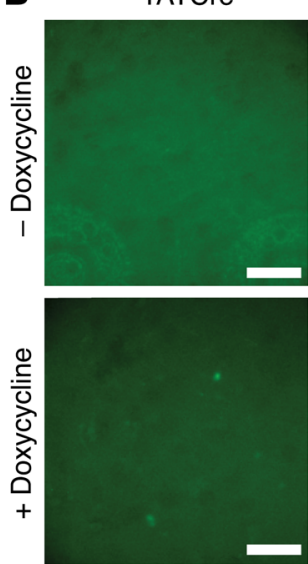

D
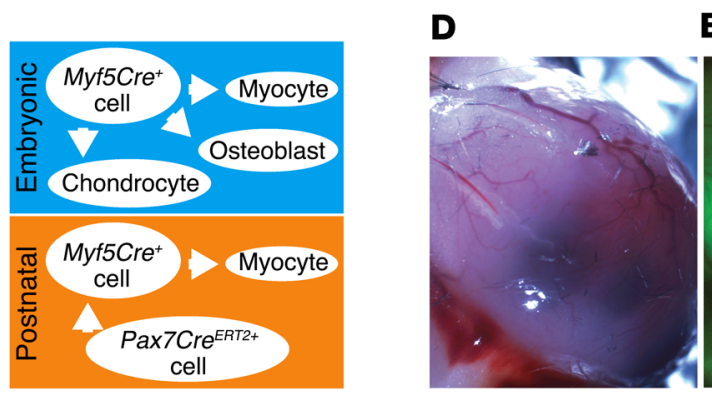

+ TATCre
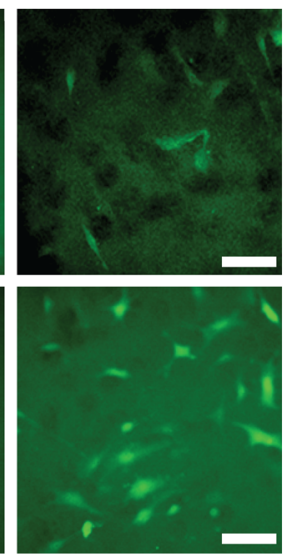

$\mathbf{E}$

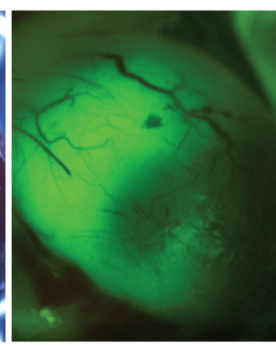

Figure 3. Synovial sarcomagenesis induced by Myf5Cre is restricted to its embryonic, multipotent lineage, not postnatal muscle progenitors. (A) Schematic of inducible alleles in mice, which depend on Cre-mediated recombination of the rtTA and the TetO promoter-controlled SS18-SSX2 allele (hSS2T), plus the presence of doxycycline for expression of the fusion oncogene. (B) Fluorescence photomicrographs showing some GFP from the recombination of $r$ TA and more GFP from the activation of $h S S 2 T$ in embryonic fibroblasts in culture. Scale bars: $100 \mathrm{uM}$. (C) Schematic and Kaplan-Meier plots of the absence of tumorigenesis in 5 groups with noted genotypes and doxycycline or postnatal induction with tamoxifen as well as rates of tumorigenesis in 2 groups with embryonic induction of SS18-SSX2 expression ( $n=25$ per group; log-rank test $Z$ score $=4.48$ aænd $P<0.001$, comparing $2 \mathrm{mg}$ and $1 \mathrm{mg}$ dosing). (D) Gross image, (E) GFP fluorescence image, and (F) H\&E histology photomicrographs of a limb tumor that developed in a 6-month-old Myf5Cre hSS2T mouse after embryonic initiation of doxycycline $(1 \mathrm{mg} / \mathrm{ml})$. Open arrowheads indicate biphasic histologic features of

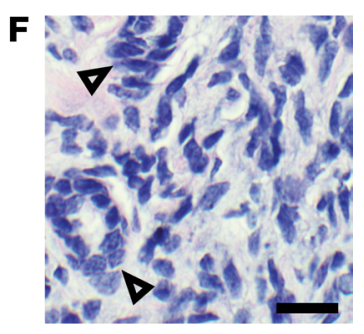
mucinous gland formation. Scale bar: $100 \mu \mathrm{m}$.

doxycycline exposure (Figure 3C). Of the 25 mice born under conditions of $1 \mathrm{mg} / \mathrm{ml}$ embryonic exposure, 2 formed tumors during the year of monitoring (Figure 3, C-F). These data suggest that the postnatal Myf5Cre lineage does not retain the SS-originating potential that it demonstrates embryonically and that myoblasts specifically may not harbor strong SS-originating potential.

To test the SS-originating potential of muscle progenitors 1 step earlier along the postnatal myogenic differentiation pathway, we induced SS18-SSX2 expression in the Pax7 lineage by tamoxifen dosing on day 5 or 8 of life in 2 cohorts of Pax7Cre ${ }^{E R T 2+} h S S 2^{+}$ mice. None of these mice developed tumors over the course of 1 year of monitoring (Figure 3C). Of note, while the myogenic precursors defined by the postnatal Myf5Cre or Pax $7 \mathrm{Cr} e^{\text {ERT2 }}$ lineages showed no significant origination potential for SS from expression of SS18-SSX2, prior data from localized, lineage-nonspecific induction of $h S S 1$ or $h S S 2$ expression had already demonstrated that some postnatal cells in both periosteal and muscle compartments retained origination potential $(5,18)$.

SS18-SSX2 expression is tolerated by differentiating osteoblast precursors and transforms the more primitive of these. The nonmyogenic fraction of differentiated cell types marked from lineage tracing after embryonic expression of $M y f 5 C r e$ suggested that the lineage includes some degree of osteochondroprogenitor differentiation capacity (8). To evaluate osteochondroprogenitors for their SS-originating potential, we conditionally activated the $h S S 2$ allele at various stages of osteoblast differentiation (Figure 4A). SS18-SSX2 expression in $\mathrm{OcCre}^{+}$cells (mature osteoblasts) resulted in no offspring surviving beyond the first day of life (Figure 4A). Likewise, few pups survived to genotyping at age 2 weeks and even fewer beyond weaning at age 3 weeks among the Colla1Cre $\mathrm{hSS}^{+}$ double-heterozygotes (preosteoblasts, Figure 4A) (31). The 3 pups that survived had densely ossified skeletons, but no detectable tumors in the soft tissue or the bones (Figure $4, \mathrm{~A}-\mathrm{C}$ ). These results highlight the developmental toxicity of the fusion oncoprotein in osteoblasts and preosteoblasts during embryonic development, as dense expression of SS18-SSX2 across these entire lineages drives developmental failure without tumorigenesis. This also fit with previous data demonstrating that Prx1Cre was lethal as an embryonic initiator of the $h S S 2$ allele (7).

To avoid embryonic/developmental toxicity of SS18-SSX expression across entire lineages, we turned our attention to tamoxifen-inducible Cre recombinase systems. Postnatal restriction of 


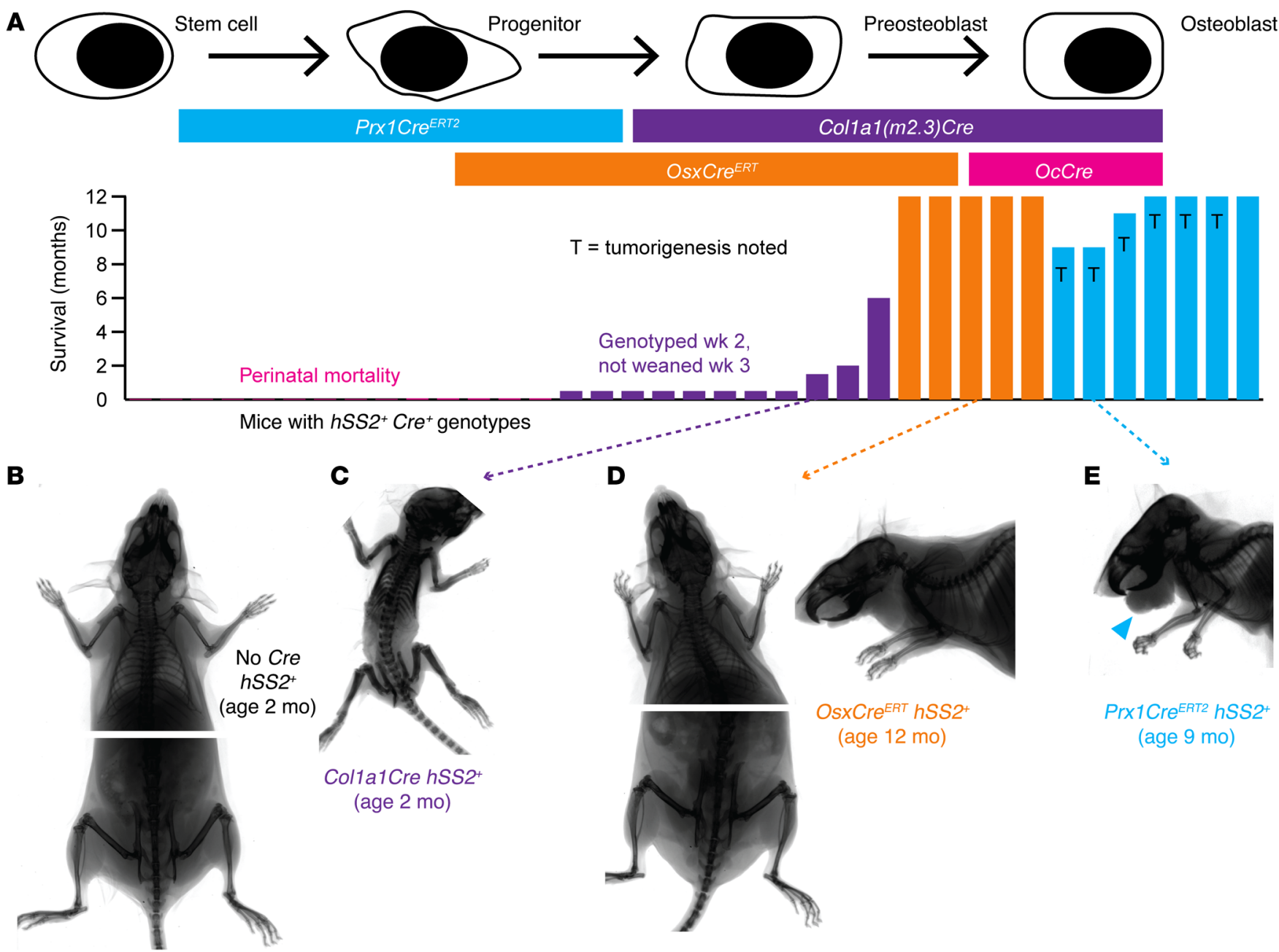

Figure 4. Impact in periosteal cells following activation of SS18-SSX2 expression. (A) Schematic of Cre drivers and survival of mice, with or without tumorigenesis following activation of $h S S 2$ at various stages of osteoblast differentiation. (B) Posteroanterior radiographs of control and (C) Colla1Cre hSS2 mice at 2 months of age, showing shortened, osteopetrotric bones in the latter. (D) Radiographs of OsxCre ${ }^{E R T} h S S 2$ mice at 12 months of age after tamoxifen administration at 4 weeks of age, showing no discernible skeletal phenotype. (E) Representative radiograph of a 9-month-old Prx1CreERT2 $h S S 2$ mouse that received tamoxifen at age 2 weeks of age and then developed the characteristic perimandibular SS (blue arrowhead).

osterix (Osx) to preosteoblasts offered the opportunity to determine whether $h S S 2$ could transform preosteoblasts without embryonic consequences $(32,33)$. After administering tamoxifen to 4-weekold OsxCre ${ }^{E R T} h S S 2$ mice, the mice were followed for 12 months and showed no sarcoma formation or any apparent skeletal phenotype (Figure 4, A and D). To induce SS18-SSX2 expression in earlier osteochondroprogenitors, we administered tamoxifen to 2-weekold Prx $1 C r e^{E R T 2}$ hSS 2 mice. Sarcomas developed in 6 of 7 mice, all on the lower lip, adjacent to the mandible (Figure 4, A and E).

Late OcCre hSS2 embryos, harvested prior to their perinatal demise, had dense bone formation and the apparent absence of hematopoietic progenitors in the marrow space (Figure 5, A and B). This niche-ablating effect has been described for the OcCre lineage in other conditional gene manipulations (24). Importantly, the spindled, marrow-replacing cells showed evidence of the recombined Rosa26 locus and expression of the fusion by the detected presence of its surrogate marker, EGFP (Figure 5, C and D). The few surviving Cola1Cre hSS2 mice also had shortened, but very dense, bones compared with those of the controls, with disorganized matrix and also evidence of continued expression of the fusion by EGFP (Figure 5, E-G). These data suggested that, among preosteoblasts, a subpopulation of cells tolerated SS18SSX2 expression without malignant transformation. Extensive prior work had not identified any such cells that expressed the fusion but did not transform. Even cells in the Myf5Cre lineage were either destroyed or gave rise to tumors, in that we found no residual Cre-recombined, $h S S 2$-expressing cells outside of the tumors in these mice.

The tumors that formed in the lower lips of Prx1Cre $e^{\text {ERT2 }}$ hSS2 mice showed strong GFP fluorescence, indicative of fusion gene expression (Figure 6, A-C). Histologic sections showed spindle-shaped cells typical of SS, with characteristic immunohistochemical profiles (Figure 6, D-F). Cross-sectional analysis of the jaw revealed the close proximity of each tumor to the bone (Figure $6 G)$. To verify the localized lineage restriction of Cre expression in the jaw/lip area, we analyzed $\operatorname{Prx} 1 \mathrm{Cr} e^{\text {ERT2 }}$ Rosa $26^{\operatorname{LacZ} Z}$ mice at 3 weeks of age, following tamoxifen administration at 2 weeks of age. The expected periosteal mesenchymal progenitor cells of the mandible 
A

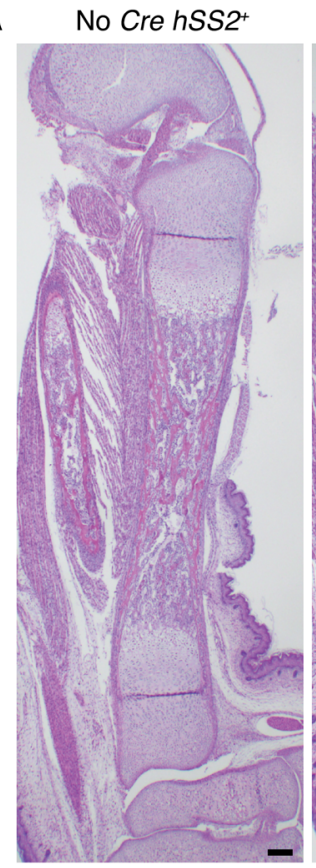

B

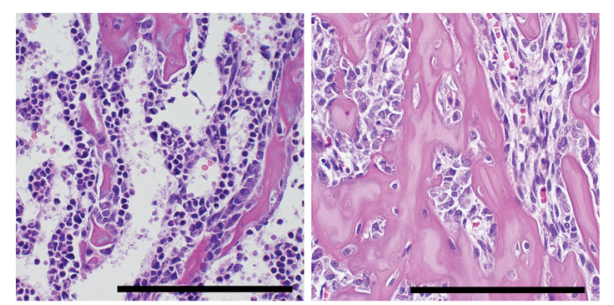

C $\mathrm{Myf5Cre}^{+} \mathrm{hSS2} 2^{+}$

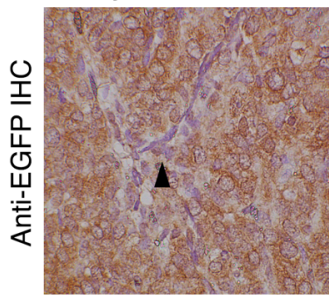

D

$\mathrm{OcCre}^{+} \mathrm{hSS2}^{+}$

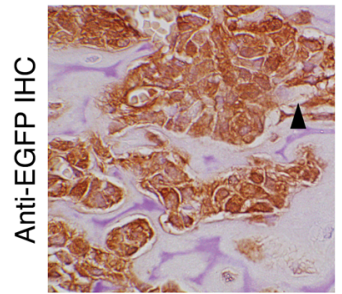

E Col1a1Cre $h S S 2^{+}$

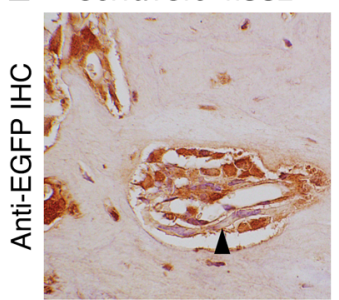

$\mathbf{F}$

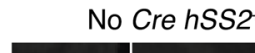

Col1a1Cre+ hSS2 $^{+}$

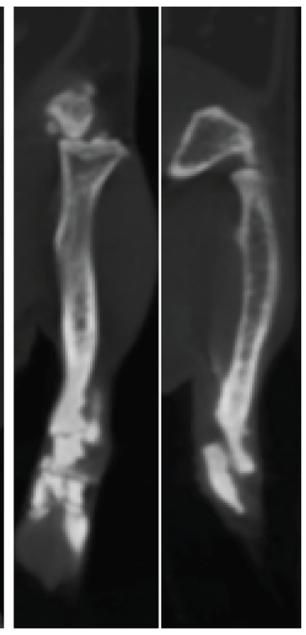

G
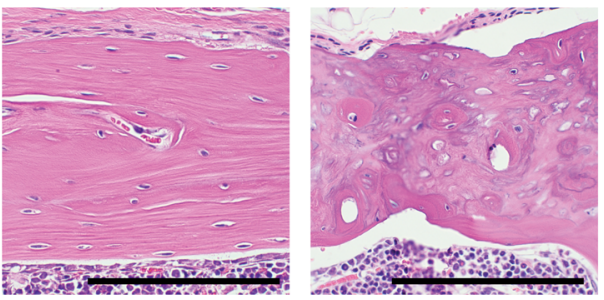

Figure 5. Pre- and perinatal osteoblasts tolerate SS18-SSX2 expression, but render dense and disorganized skeletal phenotypes. (A) H\&E histology photomicrographs of tibiae from control and OcCre hSS2 embryos harvested 18.5 days postcoitum show dense, woven bone replacing the medullary canal in the latter. (B) Higher-power photomicrographs from the same groups show the replacement of hematopoietic progenitor cells with spindle-shaped osteoblasts in the latter. Photomicrographs of anti-EGFP IHC in a Myf5Cre hSS2 tumor (C, positive control) and bone sections from (D) an OcCre hSS2 embryo and (E) the Col1a1Cre hSS2 mouse that survived to 6 months, showing fusion expression in osteoblasts in the latter 2. (F) Micro-CT axial, coronal, and sagittal images of the tibia from a control mouse and the only surviving Colla1Cre hSS2 mouse at 6 months of age, showing short, dense, and poorly remodeled bones in the latter. (C) H\&E histology photomicrographs of cortical bone from the same mice as in $\mathbf{F}$ reveal contrasting lamellar control bone in the former and disorganized ossification patterns in the latter. Scale bars (A, B, G) and panel widths: $100 \mu \mathrm{m}$ (C-F).

were the sole lineage marked in these tracing experiments (Figure $6 G)$. Notably, the adjacent enamel epithelium of the tooth has been shown to express and secrete high levels of OPG (34).

Stabilized $\beta$-catenin and SS18-SSX expression enhance sarcomagenesis in mesenchymal progenitors. Given the observed enhancement of tumorigenesis when stabilization of $\beta$-catenin accompanied localized activation of $h S S 2$ (18), we combined the $C t n n b 1^{e x 3 l}$ and $h S S 2$ alleles under the control of Prx $1 C r e^{E R T 2}$. Injection of tamoxifen into 2-week-old mice led to dramatic tumorigenesis, with the entire periosteal surface of the forelimb skeleton (the primary lineage of postnatal Prx1-expressing cells) erupting into massive tumors, all before the age of 3 months (Figure 7, A-I). Littermate control mice, lacking either $h S S 2$ expression or $\beta$-catenin stabilization, produced no tumors within a similar time frame (Figure 7A). The Prx $1 C r e^{E R T 2} h S S 2$ Ctnnbl $^{\text {exz } 3 f}$ tumors had classic SS histologic features and immunohistochemical profiles (Figure 7, J-L).

Prx1Cre ${ }^{E R T 2} C_{\text {tnnb1 }}{ }^{\text {ex3 }}$ littermates that lacked the $h S S 2$ allele showed the impact of $\beta$-catenin stabilization on the Prx1Cre ${ }^{\text {ERT2 }}$ lineage. These mice had a consistent phenotype at 1 year of age. Radiographs showed that the bones of the clavicle and forelimb had increased mineral density and thickened trabecular structure, respectively (Figure 8, A and B). Histopathology revealed an extensively thickened layer of periosteal undifferentiated mesenchyme in the forearm (Figure 8, C and D) and areas of both cartilaginous and osseous differentiation, as shown by Masson's trichrome staining (Figure 8E). Each mouse of this genotype had also developed a bland, fibrous, tumor-like thickening of the mandible (Figure 8, F and G). The fibrous jaw masses had a microscopic appearance resembling the clinical entity termed an ossifying fibroma, with encapsulated islands of osteoid and cartilaginous matrix formation, each identified by Masson's trichrome staining (Figure 8, H and I). The matrix islands contained no embedded osteocytes. Osteoblasts blocked from terminal differentiation would be excluded from the matrix they produce, as osteocyte embedding is linked to cell-cycle exit and terminal differentiation (35). The production of both osteoid and chondroid matrix con- 
A

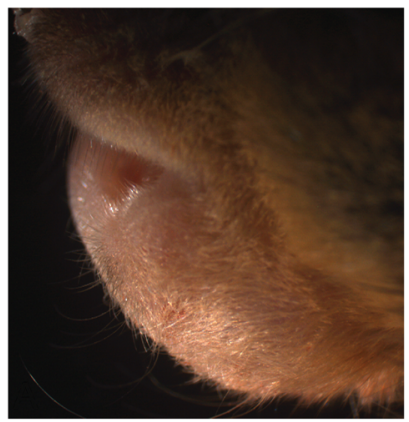

D

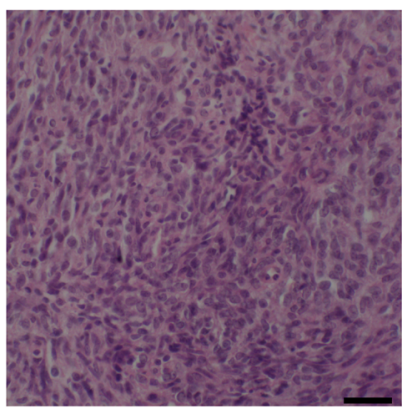

B

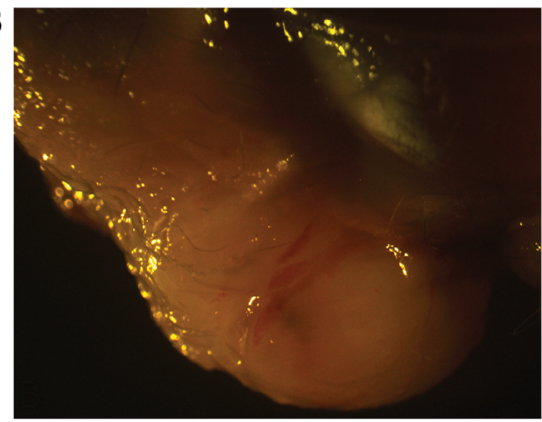

E

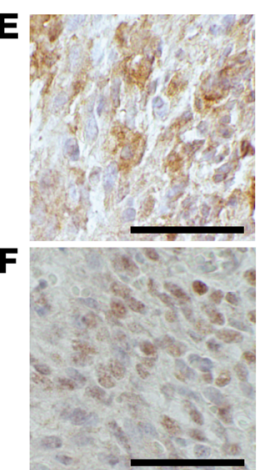

G

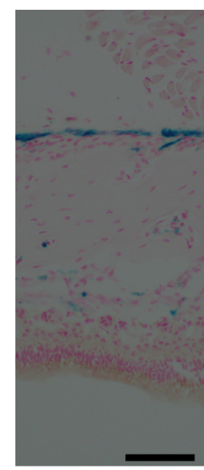

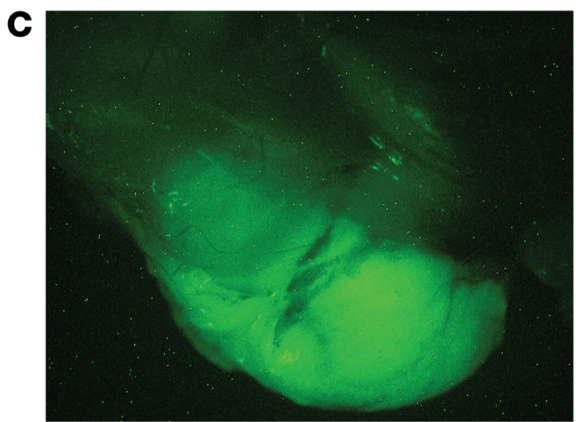

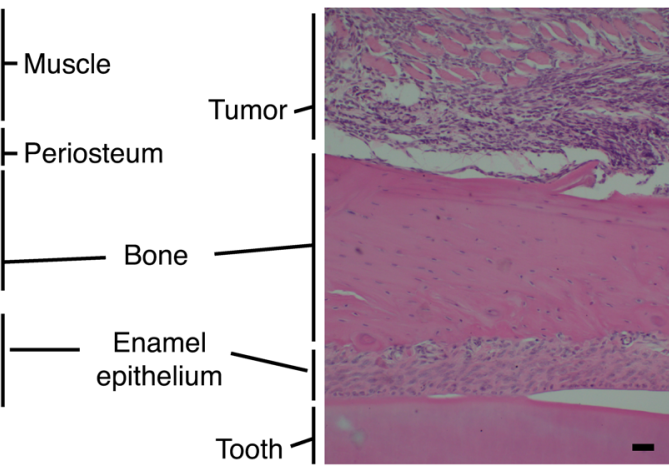

Figure 6. Expression of SS18-SSX2 in postnatal Prx1Cre ${ }^{E R T 2}$ lineage induces synovial sarcomagenesis. (A) Pre- and (B) post-dissection gross images as well as (C) a GFP fluorescence image demonstrating a representative submandibular tumor that occurred on 6 of 7 of the Prx1Cre ${ }^{E R T 2}$ hSS2 mice. (D) Representative H\&E histology photomicrograph shows typical features of a monophasic SS. Classic SS immunohistochemical staining demonstrates (E) cytoplasmic BCL2 and (F) nuclear TLE1. (G) Photomicrograph of X-gal staining of the mandible from a Prx1Cre ${ }^{E R T 2}$ LacZ mouse showing the periosteal lineage (blue, left) as well as H\&E staining of an SS tumor apparently arising from the same tissue layer in a Prx1Cre ${ }^{E R T 2}$ hSS2 mouse (right), with adjacent enamel epithelium, a strong source of OPG production. Scale bars: $50 \mu \mathrm{m}$.

firmed the extended maintenance of an incompletely differentiated state, as along the course of mesenchymal differentiation, cells retaining both osteoblastic and chondrogenic characteristics precede those committed to either lineage (36).

$\beta$-Catenin stabilization enables SS18-SSX2-mediated transformation of preosteoblasts. Given the dramatic enhancement of sarcomagenesis from the addition of $\beta$-catenin stabilization to $h S S 2$ activation in mesenchymal progenitors and the observation that this effectively blocked or slightly reversed the differentiation state of the lineage, we next tested whether such a shift along the differentiation pathway of osteoblasts could be effected in the $O s x C r e^{E R T}$ lineage by $\beta$-catenin stabilization. Mice were bred to bear heterozygosity for $C t n n b 1^{e x 3 f l}, h S S 2$, and $O s x C r e^{E R T}$ and injected with tamoxifen at 2 or 4 weeks of age. Triple-heterozygous mice also formed tumors that arose from much of the surface of the appendicular skeleton, with complete penetrance, and each tumor had classic SS histology and immunohistochemical profiles (Figure 9, A-I). Control mice, with activation of either $h S S 2$ or Ctnnb1 ${ }^{\text {xxffl }}$ alone in postnatal preosteoblasts, developed no tumors by 12 months of age (Figure 9A), but the latter allele revealed the developmental impact of $\beta$-catenin stabilization. Radiographic and histologic examination identified a fibro-osseous jaw mass in only 1 of 7 mice of this genotype (Supplemental Figure 3A). Trichrome staining revealed that the osteoid matrix islands within the fibroblastic proliferation in this mass contained no chondroid matrix (Supplemental Figure 3B). This lack of chondroid matrix correlated with the slightly later stage of osteoblast-directed dif- ferentiation at which $\beta$-catenin was stabilized in these mice, relative to the mice with $\beta$-catenin stabilization in the Prx1Cre ${ }^{\text {ERT2 }}$ lineage (compare with Figure 8I). OsxCre ${ }^{E R T} C t n n b 1^{1 \times 3 f l}$ mice also showed an increased, osteopetrosis-like density in the mid-portions of the appendicular long bones, with or without the addition of hSS2 (Figure 9, B-E). The increased density in the long bones matched the trabecular morphology and position of the primary spongiosa that would have been present in these mice at the age of 4 weeks, when tamoxifen was administered (Figure 9, J and $\mathrm{K})$, suggesting that the blockade to osteoclastogenesis was nearly complete in these portions of the bone. Bony trabeculae in these areas were thick and dense, as reflected in the area measurements from photomicrographs (Figure 9L), consistent with absent osteoclast activity. Tartrate-resistant acid phosphatase (TRAP) staining identified significantly fewer osteoclasts in these areas compared with that seen in the metaphyses of normal-bone, age-matched controls (Figure 9M).

\section{Discussion}

These data challenged our earlier interpretation that the SS-originating potential of the $\mathrm{Myf5Cre}$ lineage indicated a myoblast. We must now conclude that postnatal myoblasts and their postnatal myogenic precursors are unlikely candidates for the cell of origin implicated by the $M y f 5 C r e$ lineage. Further, exogenous OPG had a paracrine impact, enabling early growth of SS18-SSX2-transforming cells, and genetic silencing of Opg slowed synovial sarcomagenesis. Finally, early mesenchymal progenitor cells with strong 
A

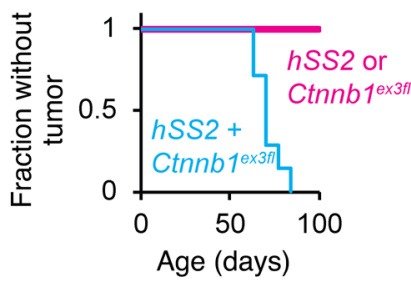

D

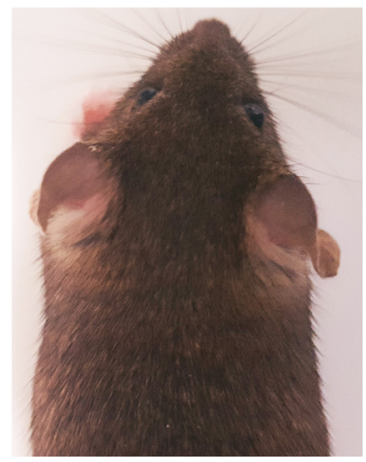

H

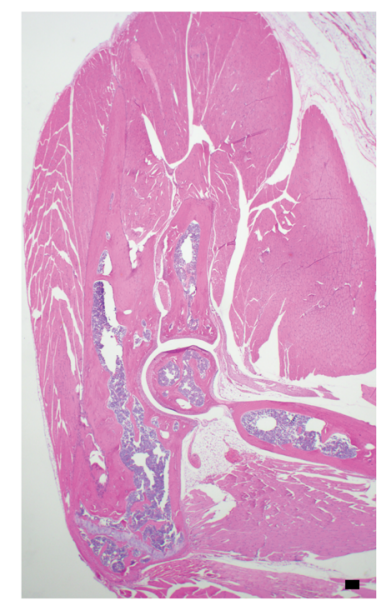

B

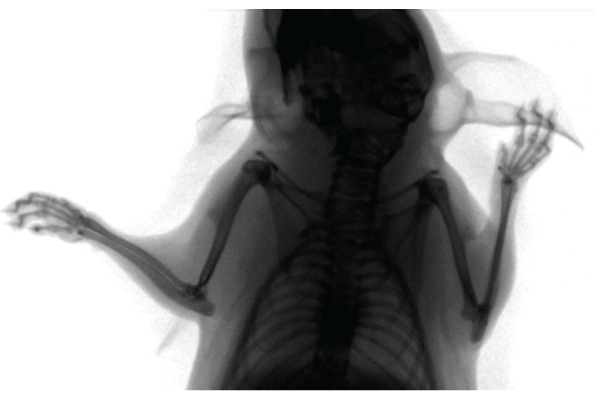

E

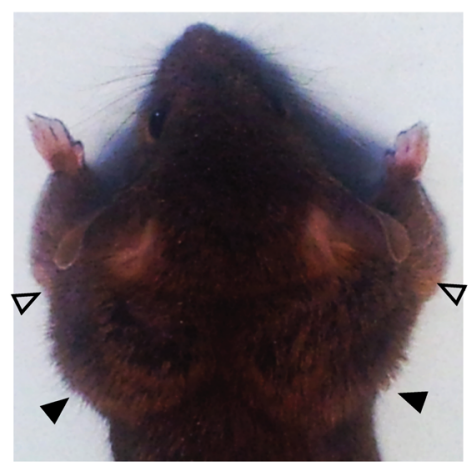

I

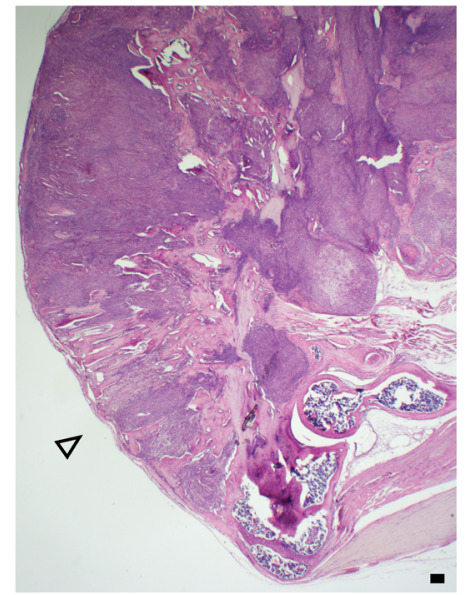

C

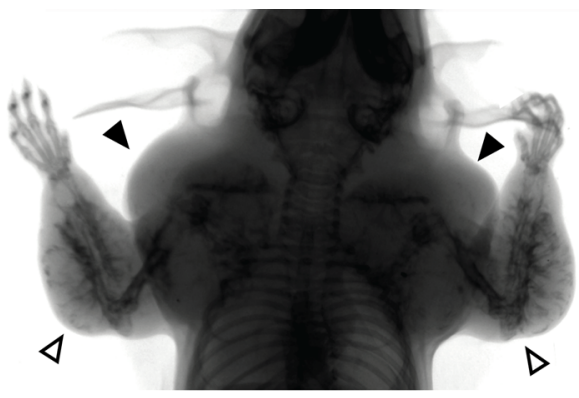

$\mathbf{F}$

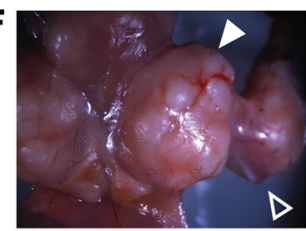

G
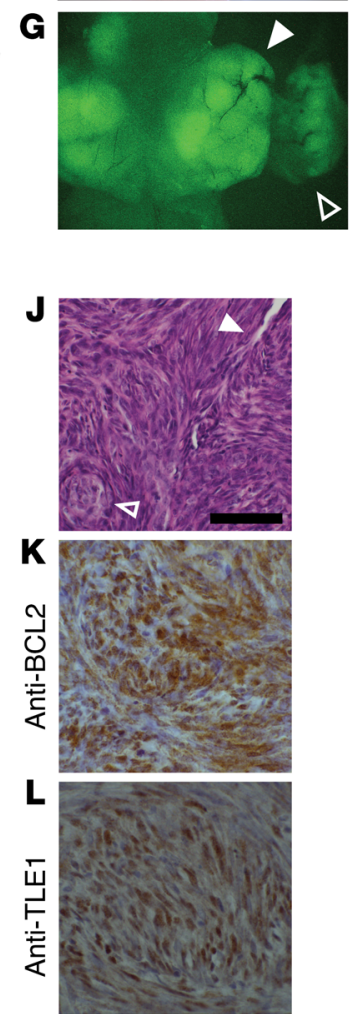

Figure 7. Stabilization of $\beta$-catenin promotes SS18-SSX2-driven transformation of mesenchymal progenitors. (A) Kaplan-Meier plot of the fraction without tumor among Prx1Cre ${ }^{E R T 2}$ mice bearing $h S S 2$, Ctnnb 1 $^{\text {ex3fl }}$, or both conditional alleles ( $n=7$ for each; $\log$ rank test $Z$ score $=3.83$ and $P<0.001$ ). (B) Posteroanterior radiographs of a control mouse and (C) a Prx1Cre ${ }^{E R T 2} h S S 2$ Ctnnb ${ }^{\text {ex3fl }}$ mouse at 10 weeks of age, as well as posteroanterior photographs of the same ( $\mathbf{D}$ and $\mathbf{E}$, respectively), showing bulky tumorigenesis in periscapular regions (solid arrowheads) and forearms (open arrowheads) of the combination genotype mouse. (F) Gross dissection photograph and (G) GFP fluorescence image of the latter mouse. (H) H\&E histology photomicrographs from sagittal sections through the elbow in a control mouse and (I) a combination genotype mouse, showing tumorigenesis from every bone surface along the ulna and radius (open arrowhead). (J) Higher-power H\&E histology photomicrograph from a tumor arising along the periosteal surface of a Prx1Cre ${ }^{E R T 2} h S S 2$ Ctnnb $^{\text {ex3fl }}$ mouse at 10 weeks of age, showing classic SS features of staghorn-style hemangiopericytomatous vessels (solid arrowhead) and pseudoglandular structures (open arrowhead), along with (K) classic immunohistochemical staining images for cytoplasmic BCL2 and (L) nuclear TLE1. Scale bars: 100 $\mu \mathrm{m}(\mathbf{H}-\mathbf{J})$; panel widths: $100 \mu \mathrm{m}$ (K and $\mathbf{L})$.

osteoblast differentiation potential proved capable of tolerating SS18-SSX2 expression and even transformation from expression of SS18-SSX2, especially with the added boost of $\beta$-catenin stabilization. Thus, the observation that human SS often arises in close proximity to bones and often has areas of osteoid matrix production within the tumor can be explained by the transforming potential in osteochondroprogenitors and by the additional enhancement of synovial sarcomagenesis from the paracrine secretion of OPG, which is highly expressed in bones. These observations also suggest an explanation of the typical age distribution for SS in adolescents and young adults. Both mesenchymal progenitor cell populations and OPG secretion diminish as humans age past young adulthood. 
A
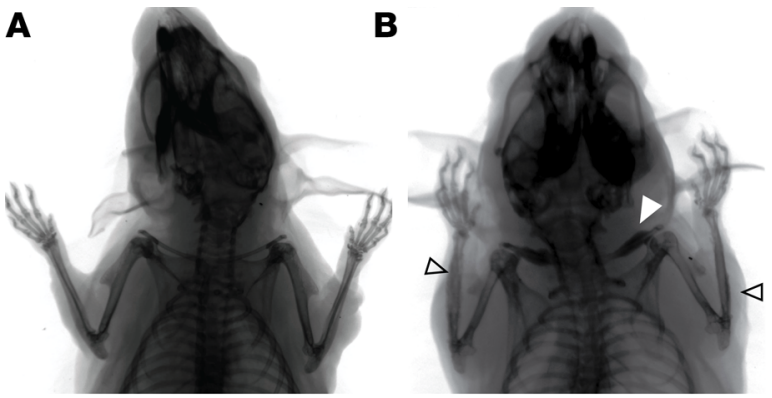

$\mathbf{F}$

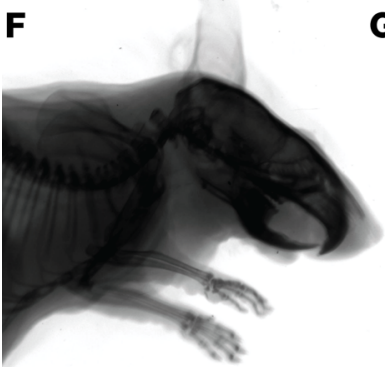

G

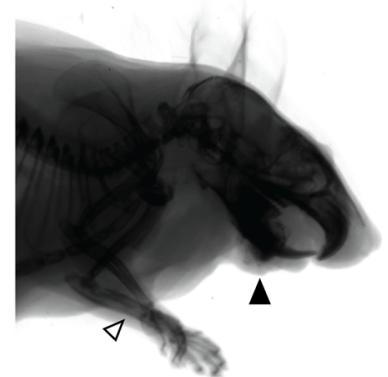

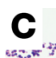

C
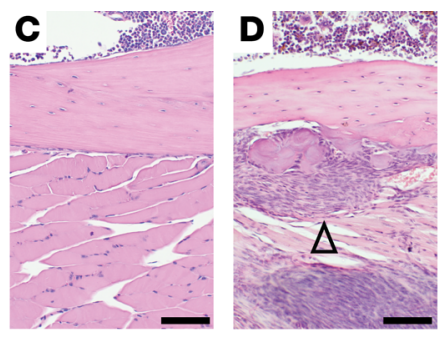

E

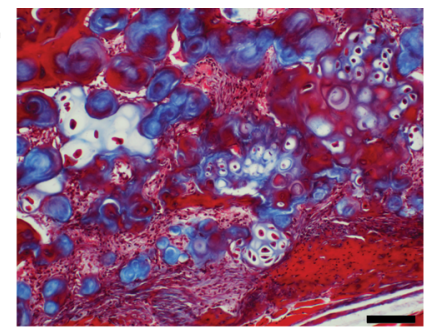

H

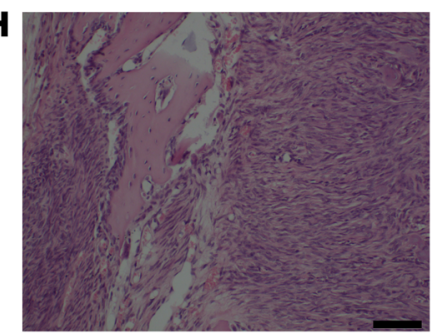

I

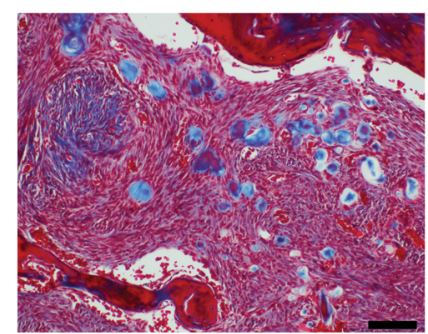

Figure 8. $\boldsymbol{\beta}$-Catenin stabilization in bone progenitors promotes proliferation and blocks differentiation. (A) Posteroanterior radiographs of a control mouse and (B) a Prx1Cre ${ }^{E R T 2}$ Ctnnb 1733fl mouse at 12 months of age show thickening of the clavicle (white arrowhead) and periosteal thickening (open black arrowheads) in the latter. (C) H\&E histology photomicrographs of the ulnar cortex from a control mouse and (D) a Prx1Cre ${ }^{\text {ERT2 }}$ Ctnnb ${ }^{\text {pex3f }}$ mouse show the thickened periosteal mesenchyme layer, which contained (E) both osseous (red) and cartilaginous (blue) matrix production on Masson's trichrome staining. Open arrowhead indicates the thickened periosteum. (F and $\mathbf{G})$ Lateral projection radiographs of a control mouse and a Prx1Cre ${ }^{\text {ERT2 }}$ Ctnnb ${ }^{\text {px3fl }}$ mouse show the same forearm periosteal reaction (open arrowhead) as well as a bone matrix-forming mass arising from the jaw (black arrowhead) in the latter. (H) $\mathrm{H} \& \mathrm{E}$-stained photomicrograph of one of these jaw masses shows a bland fibroblastic proliferation containing (I) islands of osseous (red) and cartilaginous (blue) matrix on the Masson's trichrome-stained image. Scale bars: $100 \mu \mathrm{m}$.

Mouse genetics experiments can provide proof of the sufficiency of specific combinations of cell lineages and transforming events for tumorigenesis but will never prove which cells truly give rise to similar tumor types in humans. Because the very character of each experiment results in tumorigenesis or no tumorigenesis, any untested variable may be the key between the 2 potential results. For example, elegant work by others has recently demonstrated that the sarcomagenesis-originating potential of satellite cells in particular depends on their activation in response to injury $(37,38)$. Our experiments did not address the application of this principle to synovial sarcomagenesis, but future work should. Injury response or $\beta$-catenin stabilization may be the key missing ingredient in synovial sarcomagenesis from postnatal muscle precursors, just as $\beta$-catenin stabilization appears to enable some more-differentiated periosteal cells of origin.

Mouse genetic experiments can also offer information regarding comparative efficiency, which, in the case of both the Prxl and Osx postnatal lineages, was the strongest seen in any model we have developed thus far. The addition of $\beta$-catenin stabilization to each of these originating lineages enabled both initiation (apparent by the additional numbers of tumors forming in each model) and progression (apparent by the rapid development of these tumors). Notwithstanding, these data cannot make a compelling argument that periosteal osteochondroprogenitors are the cells of origin for human SS, as some human SSs arise in anatomic locations distant from the skeleton (such as primary pulmonary SSs); the efficiency data can only suggest that conditions may be suitable for such cells to potentially serve as a frequent cell of origin for SS case.
Finally, mouse genetic experiments can test the necessity of specific genes for transformation by their removal from otherwise efficient models of tumorigenesis. Such negative genetic experiments may be performed in the transforming cells or in the surrounding cells. By using a nonconditional germline mutant allele for Opg loss of function, we did not distinguish between these two. Therefore, both tumor cell-autonomous effects of lost OPG and lost paracrine OPG from host tissues, such as bone, were combined in these experiments. Because few human SSs express OPG directly, we assumed that we were testing the paracrine impact from nontumor cells. Nonetheless, we cannot exclude the impact of cell-autonomous effects of lost OPG, because the tumor cells also lacked functional $O p g$.

SS has been termed a stem cell malignancy (39). The stem-like state of SS cells may not derive entirely from a stem-like cell of origin, as even preosteoblasts gave rise to SSs with the appropriate inducement. Some level of differentiation must be reversible through the transformation process. If a periosteal cell requires no "second hit" more drastic than stabilization of $\beta$-catenin to render synovial sarcomagenesis efficient, it may achieve that stabilization in a variety of ways. $A P C$-inactivating and $\beta$-catenin-stabilizing mutations have been described in approximately $20 \%$ of human SSs (19-22), but many other genetic, epigenetic, paracrine, and microenvironmental forces converge upon $\beta$-catenin stabilization. These alternate mechanisms of $\beta$-catenin stabilization are likely at work in a large portion of the poor-prognosis SSs with aberrant staining patterns for $\beta$-catenin. They may also provide alternate means of enabling SS18SSX-driven oncogenesis in cells as differentiated as preosteoblasts. 

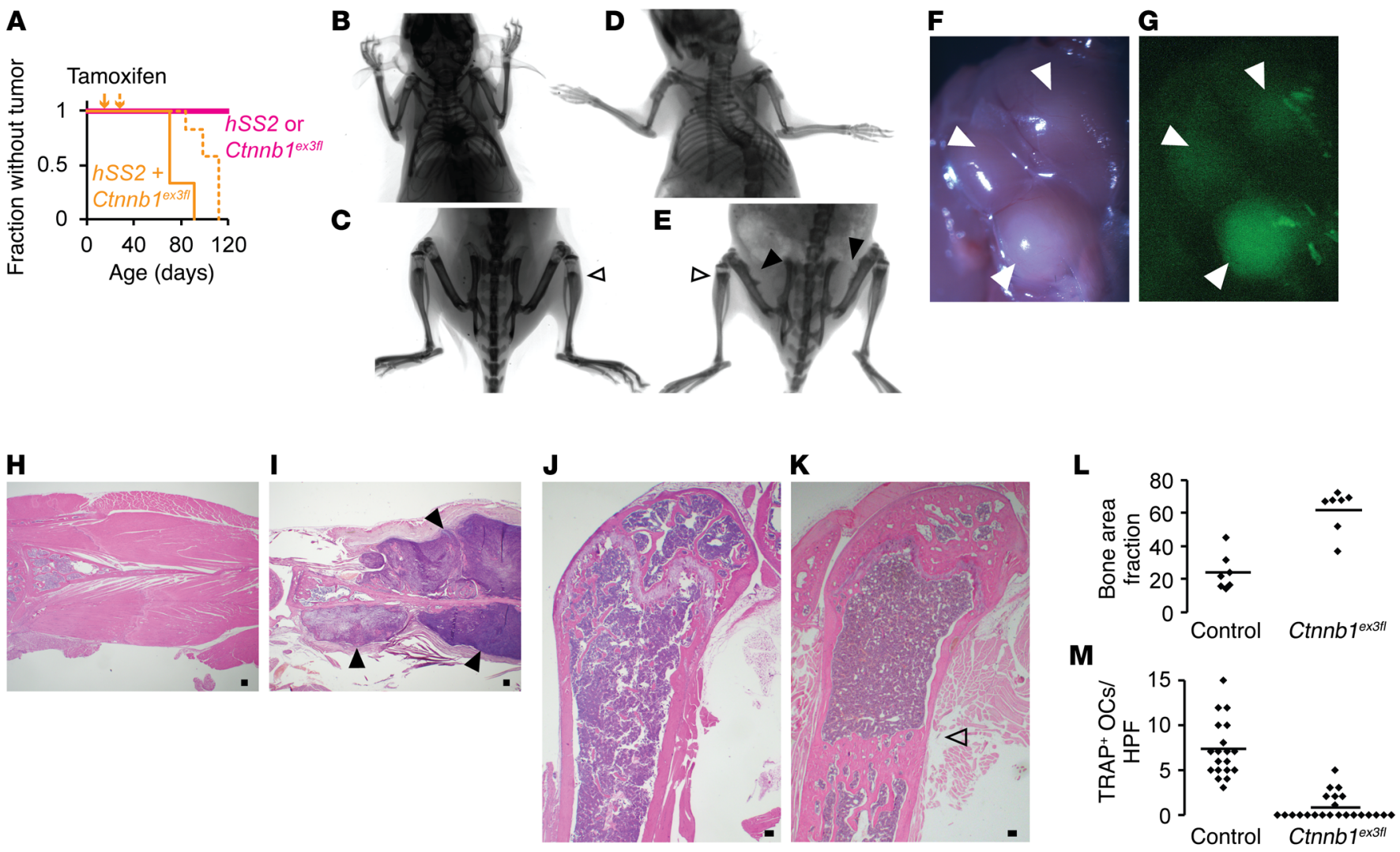

Figure 9. $\boldsymbol{\beta}$-Catenin stabilization enables synovial sarcomagenesis from preosteoblasts. (A) Kaplan-Meier plot of tumorigenesis among $0 s \times C r e^{E R T}$ mice bearing hSS2, Ctnnb ${ }^{\text {exzffl, }}$ or both and that received tamoxifen at 2 weeks of age (solid line; $n=7$; log rank test vs. control $Z$ score $=3.15$ and $P=0.0016$ ) or 4 weeks of age (dotted line; $n=7 ; Z$ score $=3.85$ and $P<0.001$ ). (B and $\mathbf{C}$ ) Posteroanterior radiographs of OsxCre ${ }^{E R T} C$ tnnb pxifl $^{\text {mice }}$ at 12 months of age, showing increased skeletal density, especially in apparently residual primary spongiosal regions, now in the metadiaphysis (open arrowhead). (D and E) OsxCre ${ }^{E R T} h S S 2$ Ctnnb ${ }^{\text {ex3fl }}$ mice at age 12 weeks of age with the same findings (open arrowhead) plus periosteal reactive bone and soft-tissue shadows (solid black arrowheads). (F) Gross

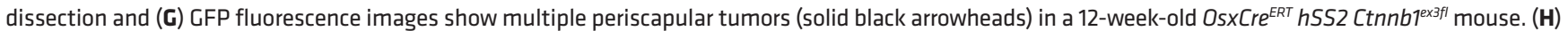
Scapular H\&E photomicrographs from a control mouse and (I) a OsXCre ${ }^{E R T} h S S 2$ Ctnnb fexif $^{\text {mol }}$ mouse, the latter showing tumors (solid black arrowheads). (J) Photomicrographs of H\&E staining of femur sagittal sections from a control mouse and (K) a OsxCre ${ }^{E R T}$ Ctnnb $1^{\text {pxifl }}$ mouse, the latter showing dense, unremodeled primary

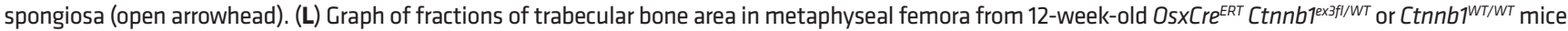
(bars depict the mean; $n=7$ femora; $P<0.0001$, by 2-tailed Student's $t$ test). (M) Graph of TRAP' osteoclasts (OCs) per high-power field (HPF) (bars depict the mean; $n=4$ femora per group and $\geq 5$ areas counted per femur; $P<0.0001$, by 2-tailed Student's $t$ test). Scale bars: 100 um.

\section{Methods}

Mice. The Rosa26 $6^{\text {hSs }}$ and Myf5Cre mouse strains were previously generated in the laboratory (7); the $C$ tnnb $1^{\text {ex3fl }}$ mouse strain was shared by Makoto Mark Taketo (Kyoto University, Kyoto, Japan) (40); Rosa26 $6^{r T A}$ mice were purchased from The Jackson Laboratory (30); Opg-mutant mice were purchased from The Jackson Laboratory (41); the Prx1Cre ERT2 $^{\text {. }}$ mouse strain was shared by Malcolm Logan (King's College in London, London, United Kingdom) (42); the OsxCre $e^{E R T}$ mouse strain was shared by Henry Kronenberg (Massachusetts General Hospital, Boston, Massachusetts, USA) (43); Col1a1Cre mice were purchased from The Jackson Laboratory (31); and the OcCre mouse strain was shared by Thomas Clemens (Johns Hopkins University, Baltimore, Maryland, USA) (44).

The $h S S 2 T$ allele was targeted to the 3 '-UTR region of the RNAPolII locus in R1 embryonic stem (ES) cells. G418-resistant clones were screened by long-range PCR and Southern blotting to confirm full-length integration. One successfully targeted clone was injected into blastocysts. High-percentage chimeric male mice were bred, and F1-generation pups were genotyped again by long-range PCR and Southern blotting and then by standard Rosa26 PCR genotyping thereafter.
For all comparison cohort experiments, littermate controls were used to mitigate any impact from the mixed SvJ and $\mathrm{Bl} / 6$ background. TATCre injections were $10 \mu \mathrm{l}$ of a $42-\mu \mathrm{M}$ concentration. rmOPG-Fc (R\&D Systems) dissolved in saline vehicle was injected $(2.5 \mathrm{mg} / \mathrm{kg}$ ) into each anterior leg compartment every 48 hours. Doxycycline was mixed at the noted concentrations into $5 \%$ sucrose drinking water that was protected from light and changed every 72 hours. Caliper measurements of tumors were performed weekly in the proximal-to-distal and medial-to-lateral dimensions of the palpable hind limb tumors, and then the volumes were calculated by multiplying the longer dimension by the square of the shorter dimension, divided by 2 .

Clinical. Consecutive SS patients were identified from a diagnostic search through pathology archives and included only if the molecular diagnosis was confirmed and if an MRI of the primary tumor prior to surgery was available. Soft-tissue sarcoma samples and MRIs were consecutively collected from control patients and confirmed not to have synovial histopathologic features.

Imaging. Radiographs of sedated mice were obtained using a Kodak Carestream 4000 Pro Fx imaging machine (Carestream Health Inc.). 
Gross light and fluorescence images were obtained postmortem with a Leica AF6000 dissecting microscope (Leica Microsystems). Light microscopy was performed and images digitally photomicrographed using an Olympus BX43 microscope and a DP26 camera (Olympus America). CT images were acquired using an Inveon Trimodality PET/ SPECT/CT Scanner (Siemens Preclinical Solutions). CT images consisting of 360 degrees and 540 projections were acquired first. The exposure time was 1.5 seconds, with detector settings at $80 \mathrm{kVp}$ and $250 \mu \mathrm{A}$. Data were reconstructed onto a $1,056 \times 1,056 \times 1,728$ image matrix using the COBRA software package (Exxim Computing Corp.). Consequently, the effective image voxel size was $47.1 \mu \mathrm{m}$ (isotropic). Reconstructed images were analyzed and visualized using 3DSlicer, version 4.6.2 (45).

Trabecular area and GFP area measurements were performed using Photoshop (Adobe) to select and mark color-defined areas, and then Image (NIH) to measure threshold areas.

Histology. Tissues were harvested postmortem, fixed in $4 \%$ paraformaldehyde overnight, decalcified (if including bone) in $14 \%$ ethylenediaminetetracetic acid at $\mathrm{pH} 7.4$ for 2 weeks at $4^{\circ} \mathrm{C}$, and embedded in paraffin following serial dehydration in ethanol, or embedded and frozen in OCT (Sakura Finetek) following serial glucose gradient dehydration for X-gal staining. In addition to standard H\&E staining, IHC was also performed for mouse TLE1 (1:50 dilution; clone M101; Santa Cruz Biotechnology); BCL2 (1:500 dilution; clone mw-26; Santa Cruz Biotechnology); and EGFP (1:500 dilution; catalog OSE00003G; Thermo Fisher Scientific) using an IgG-HRP goat anti-rabbit secondary antibody (1:5,000 dilution; sc-2004; Santa Cruz Biotechnology) and counterstained with hematoxylin. TRAP cytochemical staining was performed by incubating slides at $37^{\circ} \mathrm{C}$ for 1 hour in phosphate-buffered $4 \%$ TRAP substrate solution (ARUP) and $0.016 \%$ pararosaniline and sodium nitrite, followed by hematoxylin counterstaining.

Statistics. The statistical analyses pertinent to each experiment are noted in the figure legends, including sample sizes. Statistical analyses were performed using a 2-tailed Student's $t$ test, with a $P$ value of less than 0.05 considered significant.

Study approval. All mouse experiments were conducted with the approval of the IACUC of the University of Utah and in accordance with international legal and ethical standards. Clinical analyses were performed with approval from the IRB of the University of Utah and in accordance with legal and ethical standards. As only extant records were reviewed, the requirement for consent was waived by the IRB.

\section{Author contributions}

KBJ and MLH conceived the experiments. JJB, BEI, HJ, MLH, YW, $\mathrm{MH}$ and $\mathrm{KBJ}$ performed experiments. JJB, AG, and KBJ analyzed data. MRC contributed mice and resources. KBJ wrote the manuscript, and all authors edited it.

\section{Acknowledgments}

The authors express gratitude for the histology technical assistance of Sheryl Tripp at ARUP Laboratories (University of Utah) and the CT imaging performed at the Small Animal Imaging Core Facility (University of Utah). The Sarcoma Foundation of America, the Paul Nabil Bustany Memorial Fund for Synovial Sarcoma Research, and the Damon Runyon Cancer Research Foundation provided funding for this project. This work was also partly supported by grants R01CA201396 and P30CA042014 from the National Cancer Institute. The content is solely the responsibility of the authors and does not necessarily represent the official views of the National Cancer Institute or the National Institutes of Health.

Address correspondence to: Kevin B. Jones, Huntsman Cancer Institute, 2000 Circle of Hope Drive, Room 4263, Salt Lake City, Utah 84112, USA. Phone: 801.585.0300; Email: kevin.jones@ hci.utah.edu.
1. Herzog CE. Overview of sarcomas in the adolescent and young adult population. J Pediatr Hematol Oncol. 2005;27(4):215-218.

2. Ladanyi M, et al. Impact of SYT-SSX fusion type on the clinical behavior of synovial sarcoma: a multi-institutional retrospective study of 243 patients. Cancer Res. 2002;62(1):135-140.

3. Barretina J, et al. Subtype-specific genomic alterations define new targets for soft-tissue sarcoma therapy. Nat Genet. 2010;42(8):715-721.

4. el-Naggar AK, et al. Synovial sarcoma. A DNA flow cytometric study. Cancer. 1990;65(10):2295-2300.

5. Jones KB, et al. The impact of chromosomal translocation locus and fusion oncogene coding sequence in synovial sarcomagenesis. Oncogene. 2016;35(38):5021-5032.

6. Miettinen M, Lehto VP, Virtanen I. Keratin in the epithelial-like cells of classical biphasic synovial sarcoma. Virchows Arch, B, Cell Pathol. 1982;40(2):157-161.

7. Haldar M, Hancock JD, Coffin CM, Lessnick SL, Capecchi MR. A conditional mouse model of synovial sarcoma: insights into a myogenic origin. Cancer Cell. 2007;11(4):375-388.

8. Haldar M, Karan G, Tvrdik P, Capecchi MR. Two cell lineages, myf5 and myf5-independent, participate in mouse skeletal myogenesis. Dev Cell.
2008;14(3):437-445.

9. Shan T, Liang X, Bi P, Zhang P, Liu W, Kuang S. Distinct populations of adipogenic and myogenic Myf5-lineage progenitors in white adipose tissues. J Lipid Res. 2013;54(8):2214-2224.

10. Deshmukh R, Mankin HJ, Singer S. Synovial sarcoma: the importance of size and location for survival. Clin Orthop Relat Res. 2004;(419):155-161.

11. Kransdorf MJ. Malignant soft-tissue tumors in a large referral population: distribution of diagnoses by age, sex, and location. AJR Am J Roentgenol. 1995;164(1):129-134.

12. Kransdorf MJ, Meis JM. From the archives of the AFIP. Extraskeletal osseous and cartilaginous tumors of the extremities. Radiographics. 1993;13(4):853-884.

13. Hara S, Hatori M, Hosaka M, Komatsu T, Tsuchiya T, Kimura N. Synovial sarcoma with massive ossification--a case report. Ups J Med Sci. 2003;108(2):151-158.

14. Hisaoka M, et al. Ossifying synovial sarcoma. Pathol Res Pract. 2009;205(3):195-198.

15. Milchgrub S, Ghandur-Mnaymneh L, Dorfman HD, Albores-Saavedra J. Synovial sarcoma with extensive osteoid and bone formation. Am J Surg Pathol. 1993;17(4):357-363.

16. Ud Din N, Hopkins A, Memon A, Ahmad Z, Ahmed R. Calcifying/ossifying synovial sarco- ma: a clinicopathologic and molecular study of 5 cases. Indian J Pathol Microbiol. 2015;58(1):55-58.

17. Winnepenninckx V, et al. Calcifying/ossifying synovial sarcoma shows $\mathrm{t}(\mathrm{X} ; 18)$ with SSX2 involvement and mitochondrial calcifications. Histopathology. 2001;38(2):141-145.

18. Barrott JJ, et al. $\beta$-catenin stabilization enhances SS18-SSX2-driven synovial sarcomagenesis and blocks the mesenchymal to epithelial transition. Oncotarget. 2015;6(26):22758-22766.

19. Saito T, et al. APC mutations in synovial sarcoma. JPathol. 2002;196(4):445-449.

20. Saito T, et al. Prognostic value of the preserved expression of the E-cadherin and catenin families of adhesion molecules and of betacatenin mutations in synovial sarcoma. JPathol. 2000;192(3):342-350.

21. Subramaniam MM, Calabuig-Fariñas S, Pellin A, Llombart-Bosch A. Mutational analysis of E-cadherin, $\beta$-catenin and APC genes in synovial sarcomas. Histopathology. 2010;57(3):482-486.

22. Iwao K, Miyoshi Y, Nawa G, Yoshikawa H, Ochi T, Nakamura Y. Frequent beta-catenin abnormalities in bone and soft-tissue tumors. Jpn J Cancer Res. 1999;90(2):205-209.

23. Glass DA, et al. Canonical Wnt signaling in differentiated osteoblasts controls osteoclast differentiation. Dev Cell. 2005;8(5):751-764. 
24. Holmen SL, et al. Essential role of beta-catenin in postnatal bone acquisition. J Biol Chem. 2005;280(22):21162-21168.

25. Lacey DL, et al. Osteoprotegerin ligand is a cytokine that regulates osteoclast differentiation and activation. Cell.1998;93(2):165-176.

26. Simonet WS, et al. Osteoprotegerin: a novel secreted protein involved in the regulation of bone density. Cell. 1997;89(2):309-319.

27. Emery JG, et al. Osteoprotegerin is a receptor for the cytotoxic ligand TRAIL.J Biol Chem. 1998;273(23):14363-14367.

28. Nakayama R, et al. Gene expression analysis of soft tissue sarcomas: characterization and reclassification of malignant fibrous histiocytoma. Mod Pathol. 2007;20(7):749-759.

29. Mitrovic D. Development of the diarthrodial joints in the rat embryo. Am J Anat. 1978;151(4):475-485.

30. Belteki G, et al. Conditional and inducible transgene expression in mice through the combinatorial use of Cre-mediated recombination and tetracycline induction. Nucleic Acids Res. 2005;33(5):e51.

31. Dacquin R, Starbuck M, Schinke T, Karsenty G. Mouse alpha1(I)-collagen promoter is the best known promoter to drive efficient Cre recombinase expression in osteoblast. Dev Dyn. 2002;224(2):245-251.

32. Maes C, et al. Osteoblast precursors, but not mature osteoblasts, move into developing and fractured bones along with invading blood vessels. Dev Cell. 2010;19(2):329-344.

33. Zhou X, et al. Multiple functions of Osterix are required for bone growth and homeostasis in postnatal mice. Proc Natl Acad Sci U S A. 2010;107(29):12919-12924.

34. Ohazama A, Courtney JM, Sharpe PT. Opg, Rank, and Rankl in tooth development: co-ordination of odontogenesis and osteogenesis. J Dent Res. 2004;83(3):241-244.

35. Uchihashi K, Aoki S, Matsunobu A, Toda S. Osteoblast migration into type I collagen gel and differentiation to osteocyte-like cells within a self-produced mineralized matrix: a novel system for analyzing differentiation from osteoblast to osteocyte. Bone. 2013;52(1):102-110.

36. Zou L, et al. Molecular mechanism of osteochondroprogenitor fate determination during bone formation. Adv Exp Med Biol. 2006;585:431-441.

37. Tremblay AM, et al. The Hippo transducer YAP1 transforms activated satellite cells and is a potent effector of embryonal rhabdomyosarcoma formation. Cancer Cell. 2014;26(2):273-287.
38. Van Mater D, et al. Acute tissue injury activates satellite cells and promotes sarcoma formation via the HGF/c-MET signaling pathway. Cancer Res. 2015;75(3):605-614.

39. Naka N, et al. Synovial sarcoma is a stem cell malignancy. Stem Cells. 2010;28(7):1119-1131.

40. Harada N, et al. Intestinal polyposis in mice with a dominant stable mutation of the beta-catenin gene. ЕМВО J. 1999;18(21):5931-5942.

41. Yun TJ, et al. Osteoprotegerin, a crucial regulator of bone metabolism, also regulates B cell development and function. JImmunol. 2001;166(3):1482-1491.

42. Hasson P, Del Buono J, Logan MP. Tbx5 is dispensable for forelimb outgrowth. Development. 2007;134(1):85-92.

43. Maes C, Kobayashi T, Kronenberg HM. A novel transgenic mouse model to study the osteoblast lineage in vivo. Ann N Y Acad Sci. 2007;1116:149-164.

44. Yeo H, et al. Conditional disruption of calcineurin $\mathrm{B} 1$ in osteoblasts increases bone formation and reduces bone resorption. J Biol Chem. 2007;282(48):35318-35327.

45. Fedorov A, et al. 3D Slicer as an image computing platform for the Quantitative Imaging Network. Magn Reson Imaging. 2012;30(9):1323-1341. 